Please send trade news information and illustrations to Arveen Bajaj at the BDJ, 64 Wimpole Street, London W1G 8YS. Trade news is supplied as a service to the reader and does not imply endorsement by the BDJ. Normal and prudent research should be exercised before purchase of use of any product mentioned.

\section{TRADE NEWS}

WHAT'S NEW

\section{Benefit for gums}

Aloe Vera Gelly provides soothing and protective benefits for the gums, inner mouth and skin according to its manufacturers.

Also available is Forever Bright Aloe Vera Toothgel, a non-abrasive formula with a fresh minty taste which uses 100\% stabilised aloe vera gel to clean, nourish and strengthen teeth and gums. The aloe vera contained in all products is organically grown.

Reader response number 50

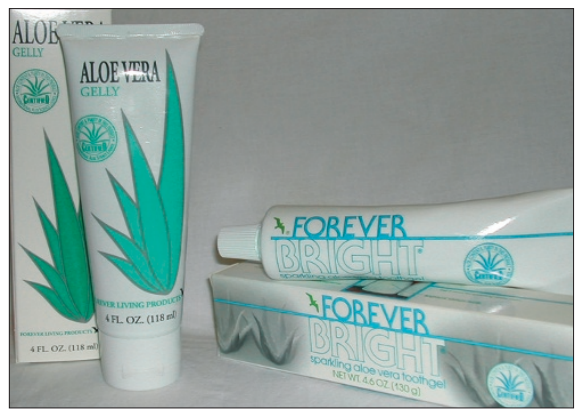

\section{Internal connection}

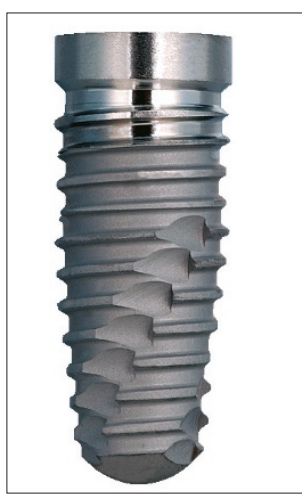

3i Implant Innovations have launched the Osseotite Certain internal connection implant system. It features QuickSeat Connection , which has an audible 'click' when placing impression copings and abutments, providing confirmation that the components are seated properly.

Its coping and abutment fingers provide retention, therefore the screw is not needed to hold the components as they are being placed.

The company claims that the product's $6 / 12$ point connection design provides flexibility for both the surgeon when placing the implant and the restorative dentist when placing the abutment.

Reader response number 51

\title{
Handpiece range expanded
}

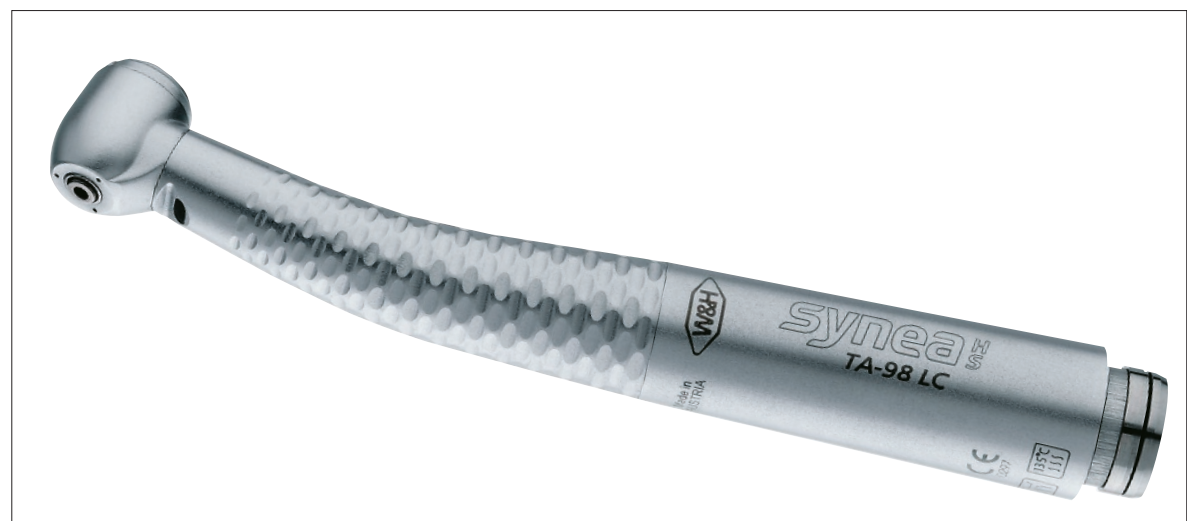

Henry Schein have introduced twelve new models of the WetH Synea HS Turbine to its range of handpieces.

According to the company, the product has to date been manufactured with integral stainless steel ball bearings with proven longevity.

Recent developments in material technology have enabled WCH to design a new range of models which incorporate integral ceramic bearings, which have a substantially longer life than integral steel bearings and carry a two year warranty. Until

now, the Synea HS range has featured only WEH Roto Quick connections, but new models are now available which couple with Multiflex giving greater flexibility within the dental practice.

Lastly modifications to the press-button chuck system have delivered a noticeable improvement in operating convenience. Optimum retention is ensured, whilst the rotary implements are now much easier to change with only a relatively light pressure on the release-button.

Reader response number 52

Patient eduCation with mouth modeller
The 3D Mouth Modeller patient edu-
cation program from MEDiVision
presents dentistry to patients in an
easy to understand format.
It enables dental treatments to be
viewed realistically from any angle
and the new Version 3 adds many
additional features.
These include a 'gearstick, which
enables each tooth to be moved into
any position to present an accurate
reflection of the real condition of
the patient's mouth. Each tooth can
be quickly drifted, over erupted and rotated. Version 3 now allows the clinician to save
each patient's settings for when treatment plans need to be re-examined or when an
illustration is required for another patient. A new zoom facility allows the zooming in
and out of areas of interest, making it possible to focus on a particular treatment area,
and provides higher definition for screen printing. It incorporates representations of a
full range of treatments and dental conditions, including four filling types, implants,
RCTs, crowns, retainers and impactions.
Reader response number 53

